\title{
Importancia de la doble exploración endoscópica (respiratoria y digestiva alta) en perros de raza bulldog francés
}

\author{
Importance of double endoscopic exploration (respiratory and digestive) in french bulldogs \\ M García-Sancho*, A Villaescusa, A Sainz, F Rodríguez-Franco
}

Departamento de Medicina y Cirugía Animal, Facultad de Veterinaria, Universidad Complutense de Madrid, Madrid, España.

\begin{abstract}
SUMMARY
The aim of this study was to evaluate endoscopic findings of respiratory and digestive tract in 18 French Bulldogs. Clinical cases included were 3 females and 15 males aged between 8 months and 5 years $(2,51 \pm 1,21)$. Clinical signs were respiratory in 3 of them (17\%) and a combination of respiratory and digestive in the other $15(83 \%) .88 \%$ presented long-term clinical signs. 13 out of 18 presented stenotic nares during physical exploration. All patients showed anomalies during the endoscopic examination of the respiratory tract (mainly, elongated soft palate, everted laryngeal saccules, congestive and edematous laryngeal cartilages and congestive trachea with mucous content) and the upper gastrointestinal tract (mainly, gastroesophageal sphincter incompetence, gastro-oesophageal reflux, oesophageal dilation and imaging suggestive of inflammation in the oesophagus, stomach and duodenum). After surgery of the upper respiratory tract anomalies, a marked improvement was obtained for both respiratory and gastrointestinal disorders in all dogs. It was concluded that the results of the present study support that routinely endoscopic respiratory and digestive exploration is of utmost importance in French Bulldogs in order to define the anomalies as a whole presented in each animal.
\end{abstract}

Key words: endoscopic findings, French Bulldog, brachycephalic.

\section{RESUMEN}

El objetivo de este trabajo se centra en la descripción de los hallazgos endoscópicos presentes en el aparato respiratorio y digestivo superior en 18 perros de raza Bulldog francés. Estos perros eran 3 hembras y 15 machos, con edades comprendidas entre los 8 meses y los 5 años (edad media de 2,51 $\pm 1,21$ años). El cuadro clínico presentado por estos animales fue de tipo respiratorio en 3 de ellos (17\%) y de tipo mixto, respiratorio y digestivo, en los 15 restantes (83\%). El 88\% presentaban este cuadro desde siempre. En 13 de los 18 perros se observó estenosis de los orificios nasales durante la exploración física. Todos los perros mostraron alteraciones durante la exploración endoscópica de las vías respiratorias (elongación de paladar blando, eversión de los ventrículos laríngeos, cartílagos laríngeos congestivos y edematosos y mucosa traqueal congestiva con mucosidad, principalmente) y del aparato digestivo superior (incompetencia del esfínter gastroesofágico, reflujo gastroesofágico, dilatación esofágica e imagen macroscópica compatible con inflamación en esófago, estómago y duodeno, principalmente). La resolución quirúrgica de las principales alteraciones anatómicas de las vías respiratorias superiores se acompañó de una evolución favorable en todos los animales, llegando a desaparecer todos los signos clínicos, respiratorios y digestivos. En conclusión, los resultados de nuestro trabajo, sostienen la importancia de realizar de forma habitual una doble endoscopia, respiratoria y digestiva superior, en los perros de raza Bulldog francés con el fin de definir de forma certera el conjunto de alteraciones presentes en cada animal.

Palabras clave: Hallazgos endoscópicos, Bulldog francés, braquicefálicos.

\section{INTRODUCCIÓN}

Las razas braquicefálicas son cada vez más populares en muchas partes del mundo. La conformación anatómica especial que presentan se relaciona con la frecuente aparición de signos respiratorios, que se denominan en su conjunto como síndrome del braquicefálico. Clásicamente, las alteraciones anatómicas más frecuentes englobadas bajo este nombre son el estrechamiento de los orificios nasales, elongación del paladar blando y eversión

Aceptado: 18.04.2013.

* Avenida Puerta de Hierro s/n 28040- Madrid, España; mercgarc@ vet.ucm.es. de los ventrículos laríngeos (Parnell 2007, Oechtering 2010). Sin embargo, la selección excesiva en cuanto a la braquicefalia ha modificado y deformado todas las vías respiratorias altas, aumentando el número de causas responsables de un estrechamiento de dichas vías (Oechtering 2010).

Además de estas alteraciones, son numerosas las alteraciones tanto anatómicas como funcionales descritas en el aparato digestivo superior de los perros de estas razas. Así, se considera que existe una mayor predisposición en algunas de estas razas a la existencia de hernias de hiato (Lorinson y Bright 1998, Tams 2003, Poncet y col 2005, Washabau 2005), esófago redundante o desviación esofágica (Woods 1978, Unzueta y col 2010), atonía del esfínter gastroesofágico (Poncet y col 2005 
y 2006), estenosis muscular pilórica por hipertrofia de fibras musculares (Peeters 1991, Guilford 1996) e hipertrofia de la mucosa pilórica (Bellenger y col 1990, Walter y Matthiesen 1993).

El Bulldog francés forma parte del grupo de razas braquicefálicas, habiendo alcanzado una altísima popularidad en España en los últimos años. Este hecho, unido a la gran frecuencia de presentación de cuadros respiratorios y digestivos en estos animales nos llevó a plantear este trabajo. Por ello, el objetivo de este estudio se centra en la descripción de los hallazgos endoscópicos presentes en el aparato respiratorio y digestivo superior en perros de raza Bulldog francés.

\section{MATERIAL Y MÉTODOS}

En este estudio se han incluido 18 perros de raza Bulldog francés en los cuales se llevó a cabo una exploración endoscópica tanto respiratoria como digestiva superior en el Hospital Clínico Veterinario Complutense de Madrid entre los años 2007 y 2011.

Para la realización de las endoscopias se utilizó un videoendoscopio Fujinon EG-270NS (de $110 \mathrm{~cm}$ de longitud y 5,9 $\mathrm{mm}$ de diámetro). Previo a la endoscopia se recomendó un ayuno de 24 horas de sólidos y de 12 horas de líquidos. La premedicación se basó en el empleo combinado de acepromacina $(0,03 \mathrm{mg} / \mathrm{kg})$ y butorfanol $(0,3$ $\mathrm{mg} / \mathrm{kg})$ en unos casos y medetomidina $(0,005 \mathrm{mg} / \mathrm{kg})$ en otros. La inducción se realizó con propofol (3-6 mg/kg) tras la oxigenación de los pacientes, siendo el isofluorano el agente responsable del mantenimiento anestésico. En cada animal se realizó en primer lugar la exploración del aparato respiratorio y posteriormente la del aparato digestivo superior. Para la endoscopia respiratoria, los pacientes eran colocados en decúbito esternal. Tras la misma, los animales eran intubados a nivel endotraqueal y situados en decúbito lateral izquierdo para la realización de la endoscopia digestiva superior. De los casos clínicos seleccionados se han recopilado datos acerca de edad, sexo, signos clínicos, hallazgos endoscópicos, tratamiento y evolución. Durante las exploraciones respiratorias se valoró principalmente la presencia de: elongación del paladar blando, eversión de los ventrículos laríngeos, cartílagos laríngeos edematosos y congestivos o mucosa traqueal congestiva con mucosidad. Asimismo, se llevó a cabo una rinoscopia caudal en todos los animales con el fin de valorar la región de las coanas posteriores. Durante las exploraciones digestivas se valoró la presencia de: dilatación de esófago, hernia de hiato, incompetencia del esfínter gastroesofágico (esfínter abierto), reflujo gastroesofágico (presencia de contenido gástrico en el esófago durante la realización de la endoscopia), imagen compatible con esofagitis, engrosamiento de los pliegues o presencia de múltiples pliegues alrededor del píloro, imagen compatible con inflamación difusa del estómago y del duodeno.

\section{RESULTADOS}

Dentro de los perros objeto de estudio, 3 eran hembras y 15 machos, con edades comprendidas entre los 8 meses y los 5 años (edad media de 2,51 \pm 1,21 años).

El cuadro clínico presentado por estos animales fue de tipo respiratorio en 3 de ellos (17\%) y de tipo mixto, respiratorio y digestivo, en los 15 restantes (83\%). El 88\% presentaban este cuadro desde siempre. Dos de los animales habían sido sometidos previamente a intervenciones quirúrgicas en relación al síndrome del braquicefálico (palatoplastia y palato y rinoplastia). Asimismo, en 13 de los 18 perros se observó estenosis de los orificios nasales durante la exploración física (figura 1). Los signos clínicos descritos son los detallados en el cuadro 1 . Todos los perros objeto de estudio mostraron alteraciones durante la exploración endoscópica de las vías respiratorias (cuadro 1) (figuras 2, 3 y 4). En el $89 \%$ de los casos se describió la existencia simultánea de, al menos, 2 alteraciones macroscópicas.

La exploración endoscópica del aparato digestivo superior mostró alteraciones en todos los perros (cuadro 1) (figuras 5,6 y 7). En el 100\% de los casos se describió la existencia simultánea de al menos 2 alteraciones macroscópicas.

La información acerca del tratamiento y la evolución posterior fue accesible en 15 de los 18 perros incluidos inicialmente en el estudio. De los mismos, el tratamiento quirúrgico fue el de elección en 13 de ellos (87\%). Las técnicas quirúrgicas utilizadas fueron: palatoplastia (1/13), palatoplastia y rinoplastia (4/13) y palatoplastia, rinoplastia y ventriculectomía (8/13). En los 2 casos restantes se optó por un tratamiento médico a base de corticoides. En cuanto a la evolución, se consideró favora-

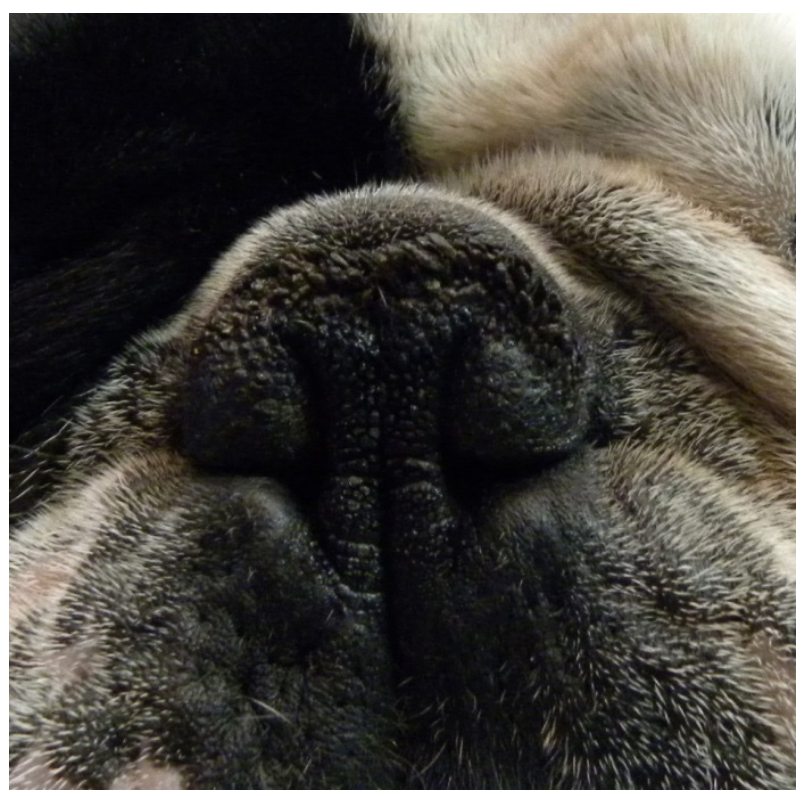

Figura 1. Estenosis de los orificios nasales. Stenotic nares. 
Cuadro 1. Signos clínicos y hallazgos endoscópicos descritos en los animales objeto de estudio. dogs under study.

Clinical signs and endoscopic findings observed in the

\begin{tabular}{lc}
\hline \multicolumn{1}{c}{ Signos clínicos } & $\begin{array}{c}\mathrm{N}^{\mathrm{o}} \text { de perros } \\
(\mathrm{n}=18)\end{array}$ \\
\hline Ronquidos y ruidos respiratorios & 18 \\
Vómitos y/o regurgitaciones & 15 \\
Intolerancia al ejercicio & 6 \\
Tos esporádica & 3 \\
Síncopes & 3 \\
Estornudos invertidos & 2 \\
Mucosas cianóticas & 2 \\
Hipersalivación & 1 \\
Diarrea & 1 \\
\hline
\end{tabular}

Hallazgos endoscópicos en

vias respiratorias

\begin{tabular}{lc}
\hline Elongación del paladar blando & 14 \\
Eversión de los ventrículos laríngeos & 11 \\
Cartílagos laríngeos congestivos y & 8 \\
edematosos & \\
Mucosa traqueal congestiva y con mucosidad & 8 \\
Mucosidad en región laríngea & 4 \\
Hipertrofia de cornetes (rinoscopia caudal) & 4 \\
Masa de pequeño tamaño en cartílagos & 3 \\
laríngeos & \\
Amígdalas engrosadas y congestivas & 2 \\
Parálisis laríngea & 1 \\
Acortamiento del paladar blando & 1 \\
\hline
\end{tabular}

Hallazgos endoscópicos en

vias digestivas altas

\begin{tabular}{lc}
\hline Incompetencia del esfínter gastroesofágico & 11 \\
Reflujo gastroesofágico & 9 \\
Dilatación esofágica & 8 \\
Engrosamiento de pliegues o & 3 \\
múltiples pliegues alrededor del píloro & \\
Hernia de hiato & 3 \\
Imagen macroscópica compatible con & 10 \\
esofagitis & 17 \\
$\begin{array}{l}\text { Imagen macroscópica compatible con } \\
\text { gastritis }\end{array}$ & $14 *$ \\
$\begin{array}{l}\text { Imagen macroscópica compatible con } \\
\text { duodenitis }\end{array}$ & \\
* La valoración endoscópica de duodeno se realizó en 16 de los 18 perros.
\end{tabular}

ble en el $100 \%$ de los casos, describiéndose una mejoría evidente de los signos clínicos (tanto respiratorios como digestivos), los cuales llegaron a desaparecer.

\section{DISCUSIÓN}

Existen estudios previos en los que se describen las alteraciones respiratorias y digestivas presentes en perros braquicefálicos (Poncet y col 2005 y 2006), siendo muy

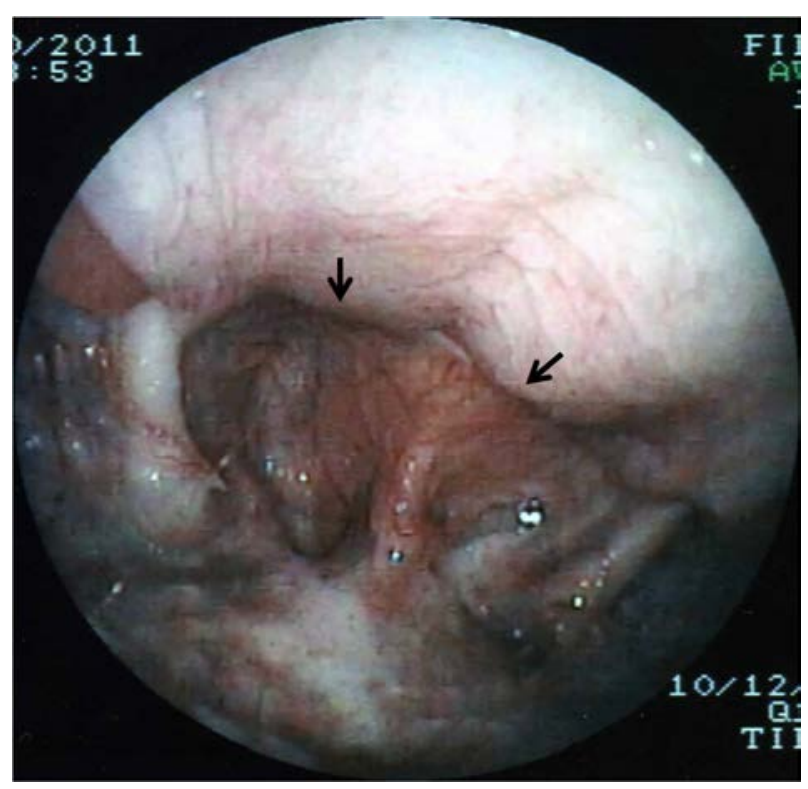

Figura 2. Elongación del paladar blando (flechas). Elongated soft palate (arrows).

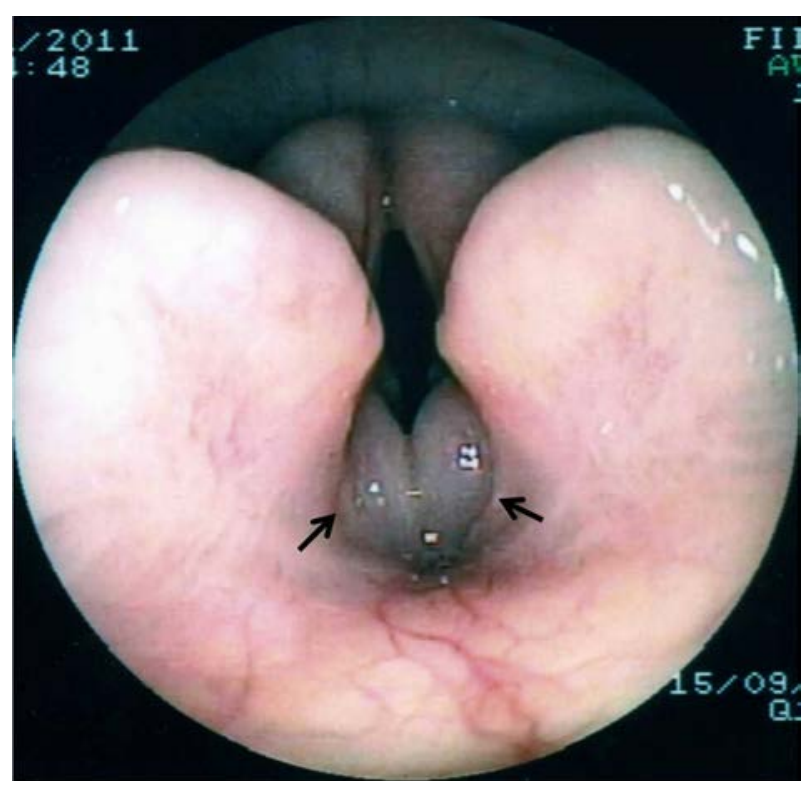

Figura 3. Eversión de los ventrículos laríngeos (flechas). Everted laryngeal saccules (arrows).

escasos los que hacen relación exclusivamente a perros de la raza Bulldog Francés (Unzueta y col 2010, García-Sancho y col 2011). Recientemente, se ha descrito la influencia del origen filogenético en el síndrome del braquicefálico, existiendo una mayor representación de razas modernas frente a las primitivas (Njikam y col 2009). Esto, unido al creciente número de ejemplares de esta raza en España, hace que sea de vital importancia caracterizar las alteraciones presentes en perros de raza Bulldog francés. 


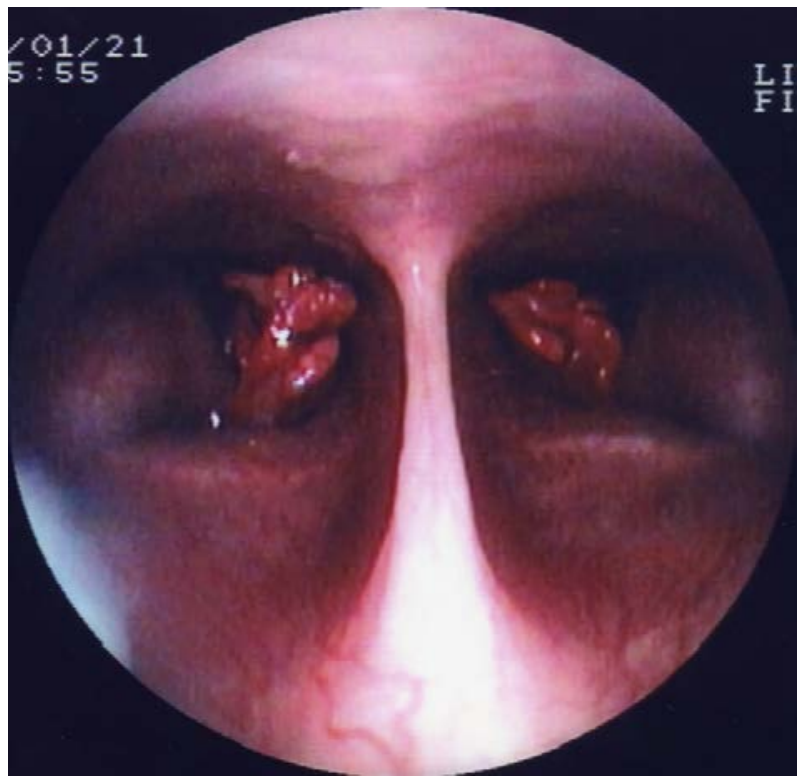

Figura 4. Imagen endoscópica de las coanas posteriores. Hipertrofia de los cornetes nasales (cornetes aberrantes caudales).

Endoscopic view of posterior choana. Abnormal conchal growth (caudal aberrant conchae).

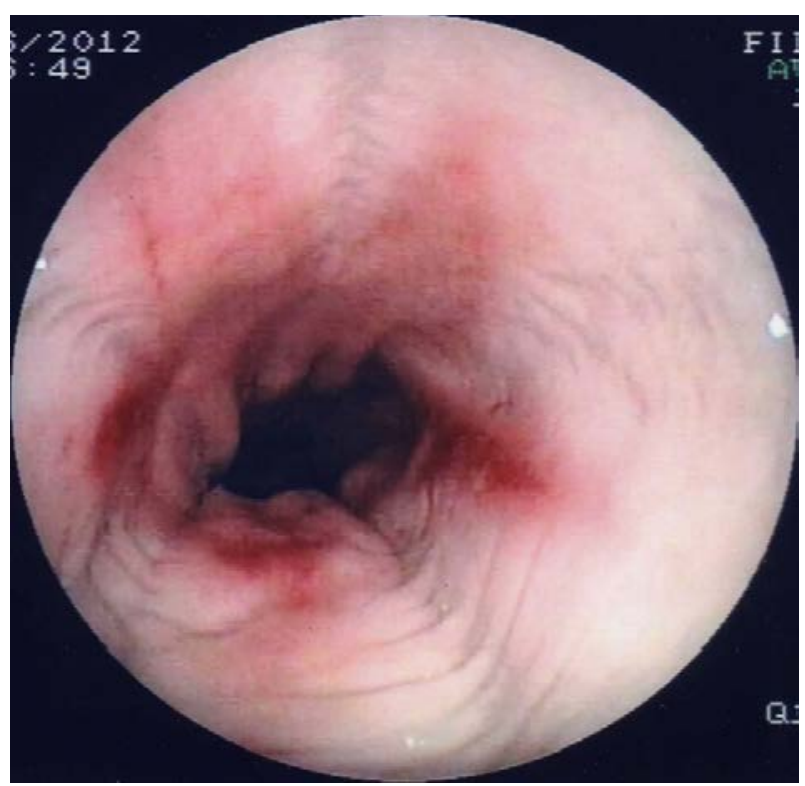

Figura 5. Mucosa enrojecida más evidente en la zona distal del esófago; imagen compatible con esofagitis. Alteración funcional a nivel del esfínter gastroesofágico el cual permanece anómalamente abierto (incompetencia del esfínter gastroesofágico).

Congestive mucosa in caudal esophagus; finding suggestive of reflux esophagitis. Functional disorder of the gastroesophageal sphincter which remains open (gastroesophageal sphincter incompetence).

Coincidiendo con otros autores, la población objeto de estudio se caracteriza por ser animales jóvenes, y fundamentalmente machos (Poncet y col 2005, 2006, Gar-

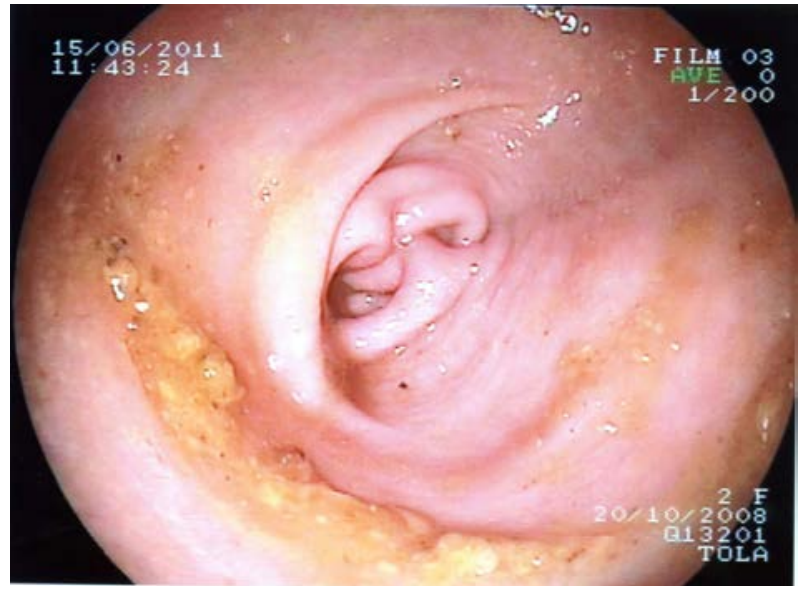

Figura 6. Imagen endoscópica de estómago. Presencia de múltiples pliegues circulares alrededor del píloro.

Endoscopic view of stomach. Multiple circular folds surrounding the pyloric lumen.

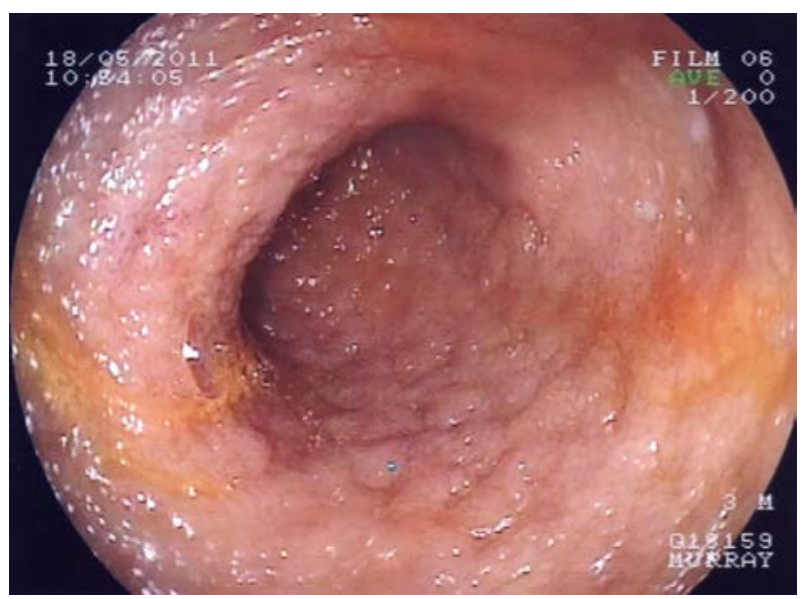

Figura 7.Imagen endoscópica de duodeno. Imagen compatible con una grave inflamación de la mucosa.

Endoscopic view of duodenum. Findings suggestive of severe inflammation.

cía-Sancho y col 2011). Hasta un $88 \%$ de estos perros presentaban signos clínicos desde siempre, hecho que, tal y como se ha sugerido previamente (García-Sancho y col 2011), podría estar relacionado con un carácter congénito de estas alteraciones. De hecho, la estenosis de los ollares, así como la elongación de paladar blando, suelen presentarse desde el nacimiento, apareciendo una clínica progresiva a medida que los perros se hacen jóvenes adultos (Trappler y Moore 2011). A pesar de la ya descrita sobrerrepresentación de los machos, no se ha propuesto ninguna hipótesis al respecto.

De los 18 perros incluidos en el estudio, la gran mayoría presentaban un cuadro mixto respiratorio y digestivo. Este hecho concuerda con trabajos previos similares 
(Poncet y col 2005 y 2006), llegándose a describir en el caso de la raza Bulldog francés una asociación estadística entre la gravedad de los signos respiratorios y los signos digestivos (Poncet y col 2005). Sin embargo, hay estudios en los que se describe una predominancia de signos respiratorios con una escasa representación de signos digestivos (Fasanella y col 2010) y viceversa, una predominancia de signos digestivos con una escasa representación de signos respiratorios (García-Sancho y col 2011). Probablemente, los criterios de inclusión aplicados en los diferentes estudios sean el motivo de estas diferencias.

Los signos clínicos más frecuentes encontrados en estos perros fueron ronquidos, ruidos respiratorios, intolerancia al ejercicio y vómitos y/o regurgitaciones, siendo estos signos los clásicamente descritos en los perros braquicefálicos (Poncet y col 2005 y 2006, Fasanella y col 2010, GarcíaSancho y col 2011). A pesar de la frecuente asociación entre sobrepeso y la gravedad de los problemas respiratorios en general, no se ha demostrado que el peso sea un factor decisivo en el síndrome braquicefálico (Riecks y col 2007).

En la línea de lo descrito por otros autores, todos nuestros perros presentaron alteraciones durante la exploración endoscópica de las vías respiratorias (Poncet y col 2005 y 2006). Es habitual encontrar en este tipo de perros al menos dos anomalías (Fasanella y col 2010), siendo la combinación de 4 de ellas lo más habitual estos perros. Nuestro estudio refleja que la elongación del paladar blando (78\%) y la eversión de los ventrículos laríngeos $(61 \%)$, junto con la estenosis de los orificios nasales (72\%), son las alteraciones más frecuentes (Oechtering 2010, Trappler y Moore 2011). Los porcentajes se encuentran entre los rangos descritos, dentro de los cuales hay variaciones justificadas por las diferencias de las poblaciones estudiadas en diferentes países.

Se ha sugerido que la elongación del paladar blando y la estenosis de los orificios nasales son componentes primarios del síndrome del braquicefálico (Poncet et al 2005). Concretamente, en el caso del paladar blando, estudios de TAC han demostrado que además existe un engrosamiento patológico añadido (Oechtering 2010). Recientemente se ha descrito que el paladar blando de las razas braquicefálicas posee unas características histológicas específicas, como es engrosamiento del epitelio superficial, edema del tejido conectivo e hiperplasia glandular en la mucosa (Pichetto y col 2011). Estos hallazgos contribuyen al engrosamiento del mismo, sin poder descartar que exista de base un defecto hereditario (Pichetto y col 2011). Sin embargo, existe cierta controversia en este punto, asumiendo algunos autores que la elongación del paladar blando podría ser una secuela de la estenosis de los orificios nasales (Aron y Crowe 1985).

En esta línea, la eversión de los ventrículos laríngeos, así como la amigdalitis se definen como cambios secundarios inducidos por aumento del esfuerzo inspiratorio y una inflamación crónica del área de la faringe (Poncet y col 2005). La frecuencia de presentación de amígdalas engro- sadas y congestivas fue muy baja (11\%), a diferencia de otros trabajos (Poncet y col 2005, Fasanella y col 2010).

Respecto a la hipertrofia de los cornetes nasales, descrita en un $22 \%$ de los perros objeto de estudio, es algo que merece la pena resaltar. Algunos autores hacen referencia a este hallazgo con el término de cornetes aberrantes rostrales o caudales (Oechtering y col 2007, Oechtering 2010), describiendo su presentación hasta en un $47 \%$ de perros de raza Bulldog francés (Oechtering y col 2007). La existencia de este tipo de cornetes provoca un aumento significativo de la resistencia intranasal al paso del aire ${ }^{1}$. Asimismo, se cree que la nariz del perro es esencial para la termorregulación eficaz, por lo que este proceso podría verse afectado en estos animales. Es probable que los trastornos relacionados con el calor, estén causados por la restricción de la regulación de la temperatura más que por la reducción de la ventilación pulmonar (Oechtering 2010). Por todo ello, algunos autores consideran que la existencia de una hipertrofia de los cornetes, así como otras posibles causas de estenosis de la cavidad nasal, debe ser valorada junto a las clásicas alteraciones del síndrome del braquicefálico (Oechtering 2010). Estos hallazgos no se describen en estudios previos realizados en perros con síndrome braquicefálico (Poncet y col 2005 y 2006, Torrez y Hunt 2006, Fasanella y col 2010). Este hecho probablemente está justificado por la ausencia de una exploración endoscópica rutinaria de esta zona, centrándose exclusivamente en la valoración del paladar y la zona de la laringe.

Todos los perros mostraron alteraciones durante la exploración endoscópica del aparato digestivo superior, al igual que se describe en la bibliografía previa (Poncet y col 2005 y 2006, García-Sancho y col 2011). De nuevo, siempre aparecen, al menos, dos alteraciones de forma conjunta (García-Sancho y col 2011). Llama nuestra atención la existencia de hallazgos endoscópicos digestivos incluso en los 3 perros cuyo cuadro clínico era exclusivamente respiratorio. Poncet y col (2005) afirman al respecto, que ningún perro con problemas respiratorios está exento de tener anomalías digestivas asociadas. Este hecho, en nuestro caso, podría estar justificado por tratarse de un estudio retrospectivo, pudiendo faltar información de las historias clínicas, o bien por no haber pasado el tiempo suficiente para desarrollar signos de tipo digestivo en respuesta a los hallazgos encontrados.

La mitad de los perros (56\%) mostraron una imagen compatible con esofagitis por reflujo, estando asociada en todos los casos a la existencia de contenido gástrico en el esófago durante la realización de la endoscopia o de un esfínter gastroesofágico incompetente. La presen-

Hueber JP, HJ Smith, P Reinhold, C Nöller, C Thiele, GU Oechtering. Brachycephalic airway syndrome: effects of partial turbinectomy on intranasal airway resistance. 2009. $25^{\text {th }}$ Symposium of the Veterinary Comparative Respiratory Society. Lafayette, Indiana, Estados Unidos. Disponible en www.brachycephalia.com. 
cia de reflujo gastroesofágico es la causa más frecuente de esofagitis en pequeños animales (Tams 2003). Se ha descrito la implicación de diversos factores en la patogenia del reflujo gastroesofágico, como es la presencia de vómito crónico, de alteraciones en el vaciado gástrico, de hernia de hiato o el efecto de la anestesia sobre el esfínter gastroesofágico (Washabau 2005). La presencia de varias de estas anomalías en los perros de raza Bulldog francés sería responsable de la imagen de esofagitis observada en una amplia mayoría de ellos.

Se observó dilatación esofágica en el $44 \%$ de nuestros pacientes, pudiendo estar este hallazgo relacionado con la existencia de regurgitaciones e hipersalivación presentes en algunos de ellos. En perros de la raza Bulldog inglés (Woods 1978, Poncet y col 2005) y francés (Poncet y col 2005, Unzueta y col 2010) se ha descrito previamente la presencia de desviaciones esofágicas o esófagos redundantes. Son esófagos de diámetro normal pero excesivamente elongados que describen una trayectoria sinuosa. En nuestros pacientes, durante la exploración endoscópica lo que se observó fueron esófagos con un diámetro mayor al normal y con una trayectoria recta, por lo que consideramos que podrían tratarse de alteraciones diferentes.

En relación a las hernias de hiato, un estudio con Bulldogs muestra que la presencia de hernias de hiato deslizantes estaba asociada a las manifestaciones más graves del síndrome braquicefálico (Hardie y col 1998). Otros trabajos describen la existencia de hernias de hiato deslizantes en perros braquicéfalos, pero no paraesofágicas (Poncet y col 2005, 2006). Las 3 hernias de hiato descritas en este trabajo, son de tipo paraesofágico. Este tipo de hernias son mucho menos frecuentes y se asocian menos al reflujo gastroesofágico, ya que se supone que el esfínter gastroesofágico permanece funcional y en una localización intraabdominal normal (Tams 2003). Por ello, es más difícil de explicar la presencia de hernias de tipo paraesofágico entre los perros de nuestro estudio, así como su implicación en los signos clínicos presentes en estos animales.

El engrosamiento de los pliegues o presencia de múltiples pliegues alrededor del píloro se observó en el $17 \%$ de los casos. Hasta el momento, la frecuencia de presentación de este hallazgo ha sido superior (Poncet y col 2005 y 2006, García-Sancho y col 2011), lo que podría ser debido a los diferentes criterios de inclusión empleados. Asimismo, se ha descrito la presencia ocasional de anillos circulares alrededor del píloro o pliegues pronunciados en el antro como variantes anatómicas normales (Tams 1990). Desconocemos si en los 3 perros que presentaron este hallazgo, cuya edad era de en torno a los 2 años, se trataba de una forma congénita presente desde el nacimiento o de una forma adquirida secundaria a la inflamación crónica y a la depresión respiratoria (Poncet y col 2005).

La imagen endoscópica evidenció la existencia de inflamación difusa a nivel gástrico en un $94 \%$ de los perros y a nivel duodenal en un $88 \%$ de los mismos, siendo este último porcentaje superior a los datos previos (Poncet y col 2005). Esto llama más la atención si recordamos que tan sólo uno de los perros objeto de estudio tenía diarrea. Se ha descrito que la imagen histológica en la mayoría de los perros braquicefálicos con signos respiratorios y/o digestivos muestra una inflamación crónica tanto a nivel de estómago como de duodeno, incluso en animales con una imagen endoscópica normal (Poncet y col 2005). Con el fin de entender mejor estos resultados, sería interesante describir las características endoscópicas e histológicas típicas de perros sanos de la raza Bulldog francés.

Finalmente, cabe resaltar la evolución favorable de todos los perros tras la resolución quirúrgica, en la mayoría de los casos, de las principales alteraciones anatómicas de las vías respiratorias superiores. Tal y como han descrito otros autores (Poncet y col 2005, 2006), la desaparición de los signos respiratorios se acompañó en todo momento de una desaparición de los signos digestivos. Asimismo, la raza Bulldog francés ha mostrado una correlación significativa entre la gravedad de los signos respiratorios y digestivos (Poncet y col 2005). Por todo ello, cada vez parece más evidente la estrecha relación entre las alteraciones respiratorias y las digestivas existentes en los perros braquicefálicos.

En conclusión, los resultados de nuestro trabajo sostienen la importancia de realizar de forma habitual una doble endoscopia, respiratoria y digestiva superior, en los perros de raza Bulldog francés con el fin de definir de forma certera el conjunto de alteraciones presentes en cada animal.

\section{REFERENCIAS}

Aron DN, DT Crowe. 1985. Upper airway obstruction: General principles and selected conditions in the dog and cat. Vet Clin N Am Small Anim Pract 15, 891-917.

Bellenger CR, JE Maddison, GC MacPherson, JE Ilkiw. 1990. Chronic hypertrophic pyloric gastropathy in $14 \mathrm{dogs}$. Aust Vet $J$ 67, 317-320.

Fasanella FJ, JM Shivley, JL Wardlaw, S Givaruangsawat. 2010. Brachycephalic airway obstructive syndrome in dogs: 90 cases (1991-2008). J Am Vet Med Assoc 237, 1048-1051.

García-Sancho M, F Rodríguez-Franco, S Canfrán, V Carrasco, A Villaescusa, A Sainz. 2011. Hallazgos endoscópicos en esófago y estómago en perros de la raza Bulldog francés. Clin Vet Peq Anim 31: 29-34.

Guilford WG, DR Strombeck. 1996. Chronic gastric diseases. In: Strombeck DR (ed). Strombeck's small animal gastroenterology. $2^{\mathrm{a}}$ nd. WB Saunders Company, Filadelphia, USA, Pp 275-302.

Hardie EM, O Ramírez, EM Clary, JN Kornegay, MT Correa, RA Feimster, ER Robertson. 1998. Abnormalities of the thoracic bellows: stress fractures of the ribs and hiatal hernia. J Vet Intern Med 12, 279-287.

Lorinson D, RM Bright. 1998. Long-term outcome of medical and surgical treatment of hiatal hernias in dogs and cats: 27 cases (1978-1996). J Am Vet Med Assoc 213, 381-384. 
Njikam IN, M Huault, V Pirson, J Detilleux. 2009. The influence of phylogenic origin on the occurrence of brachycephalic airway obstruction syndrome in a large retrospective study. Intern J Appl Res Vet Med 7, 138-143.

Parnell NK. 2007. Enfermedades de la garganta. En: Ettinger SJ, Feldman EC (eds). Tratado de Medicina Interna Veterinaria. $6^{\mathrm{a}}$ ed. Elsevier España S.A., Madrid, España, Pp 1196-1204.

Peeters ME. 1991. Pyloric stenosis in the dog: Developments in the surgical treatment and a retrospective study in 47 patients. Eur J Compan Anim Pract 2, 37-40.

Pichetto M, S Arrighi, P Roccabianca, S Romuss. 2011. The anatomy of the dog soft palate. II. Histological evaluation of the caudal soft palate in brachycephalic breeds with grade I Brachycephalic Airway Obstructive Syndrome. Anat Rec 294, 1267-1272.

Oechtering GU, JP Hueber, TH Oechtering, C Noeller. 2007. Laser assisted turbinectomie (LATE)-Treating brachycephalic airway distress at its intranasal origin. Vet Surg 36, E18.

Oechtering G. 2010. Síndrome braquicefálico - Nuevos conocimientos sobre una vieja enfermedad congénita. Vet Focus 20, 2-9.

Poncet CM, GP Dupre, VG Freiche, MM Estrada, YA Poubanne, BM Bouvy. 2005. Prevalence of gastrointestinal tract lesions in 73 brachycephalic dogs with upper respiratory syndrome. J Small Anim Pract 46, 273-279.

Poncet CM, GP Dupre, VG Freiche, BM Bouvy. 2006. Longterm results of upper respiratory syndrome surgery and gastrointestinal tract medical treatment in 51 brachycephalic dogs. J Small Anim Pract 47, 137-142.

Riecks TW, SJ Birchard, JA Stephens. 2007. Surgical correction of brachycephalic syndrome in dogs: 62 cases (19912004). J Am Vet Med Assoc 230, 1324-1328.

Tams TR. 1990. Gastroscopy. In: Tams TR (ed). Small Animal Endoscopy. Mosby, San Luis, USA, Pp 89-166.

Tams TR. 2003. Diseases of the esophagus. In: Tams TR (ed). Handbook of Small Animal Gastroenterology. $2^{\mathrm{a}}$ nd. WB Saunders Company, Philadelphia, USA, Pp 118-158.

Trappler M, KW Moore. 2011. Canine brachycephalic airway syndrome: pathophysiology, diagnosis, and nonsurgical management. Compend Contin Educ Vet 33, E1-E5.

Unzueta Galarza A, A Villegas Corrales, B Llamosas Caballero, I Luño Muniesa, O Fernandez Aguirre, AM Perez Castro. 2010. Estudio de prevalencia de esófago redundante en la raza Bulldog francés. Póster $n^{\circ} 75$ perteneciente a la sección de Gastroenterología del Southern European Veterinary Conference- 45 Congreso Nacional A.V.E.P.A. Barcelona, España.

Walter MC, DT Matthiesen. 1993. Acquired antral pyloric hypertrophy in the dog. Vet Clin North Am Small Anim Pract 23, 547-554.

Washabau RJ. 2005. Disorders of the pharynx and oesophagus. In: Hall ED, Simpson JW, Williams DA (eds). BSAVA manual of Canine and Feline Gastroenterology. $2^{\mathrm{a}}$ nd. BSAVA, Gloucester, UK, Pp 176-203.

Woods CB, C Rawlings, D Barber, M Walker. 1978. Esophageal deviation in four English Bulldogs. $\mathrm{J} \mathrm{Am} \mathrm{Vet} \mathrm{Med}$ Assoc 172, 934-939. 
